\title{
Contents, Vol. 34, 1972
}

\section{Index}

Antoni, F.: vide Piffkó, P.

Antoni, F.: vide Puskás, M.

Axelsson, A. and Brorson, J.-E.: Bacteriological Findings in Acute Maxillary

Sinusitis 1

Bernath, O.: vide Nyffenegger, R.

Bird, E. S.: vide Friedmann, I.

Boniver, R.: vide Penders, C.

Branefors-Helander, P.; Nylén, O., and Jeppsson, P.-H.: Acute Otitis Media.

A Bacteriological Study 281

Brorson, J.-E.: vide Axelsson, A.

Dammert, K.: vide Palva, T.

Donat-Jasiak, T.: vide Pruszewicz, A.

Etholm, B.: Non-Fatal Transverse Fractures of the Temporal Bone - Histo-

pathology, Late Complications

273

Friedmann, I.; Michaels, L.; Gerwat, J., and Bird, E. S.: The Microscopic

Anatomy of the Nasopharyngeal Tonsil by Light and Electron

Microscopy 195

Gerwat, J.: vide Friedmann, I.

Gy. Farkas: vide Puskás, M.

Hänninen, P.: vide Puhakka, H. J.

Hansson, C. G. and Lindström, J.: Primary Branchiogenic Carcinoma. Report

of a Case

Haubrich, J.: vide Miehlke, A.

Hoshino, T. and Ishii, D.: Intralabyrinthine Neurilemmoma. A Histopath-

ological Report

Igarashi, M.; Withers, B. T., and M $\gamma y$ ata, H.: The Distribution of L-

Thyroxine Labeled with Radioactive Iodine-125 in the Ears of

Squirrel Monkeys 240

Ishii, D.: vide Hoshino, T.

Jeppsson, P.-H.: vide Branefors-Helander, P.

John, H. D.: vide Westergaard, O.

Jong, P. C. de: The Use of $107 \mathrm{HgCl} 2$ in Diagnosis of Malignant Tumors of the Head and Neck Region. Preliminary Findings 303

Juhn, S. K.: vide Youngs, J. N.

Kallenberger, Alice; Wey, W., and Renner, K.: DNA Content and Radiosensitivity of Malignant Tumours of the Upper Respiratory and

Digestive Tract 65

Kessler, L. und Krisch, A.: Zur Topographie und Chirurgie des kongenitalen 
Kluyskens, P.: vide Westergaard, O.

Köteles, G. J.: vide Piffkó, P.

Index III

Kornblut, A. D.: Bleomycin Treatment in Patients with Inoperable Malig nancy of the Head and Neck 325

Krisch, A.: vide Kessler, L.

Kwee, H. L.: A Case of Tullio Phenomenon with Congenital Middle-Ear Ab

normalities 145

Lindström, L: vide Hansson, C. G.

Meyer, R.: Reconstruction de la columelle et de la cloison nasale (avec film) 170

Michaels, L.: vide Friedmann, I.

Miehlke, A.; Stennert, E.; Schuster, R.; Schätzle, W. und Haubrich, J.:

Über die Regeneration peripherer Nerven nach Einwirkung ionisie-

render Strahlen 88

Minnigerode, B.: Das Nystagmusbild beim synkopalen vertebro-basilären An-

fallsyndrom 101

Miyata, H.: vide Igarashi, M.

Miyoshi, T. and Pfaltz, C. R.: Studies on Optokinetic Habituation 308

Nielsen, B. Lade: Changes in the Upper Respiratory Tract after Prolonged

Naso-Tracheal Intubation 210

Nyffenegger, R. und Bernath, O.: Zum Problem des akustischen Unfalls ... 252

Nylén, O.: vide Branefors-Helander, P.

Obrebowski, A.: vide Pruszewicz, A.

Palva, T.; Palva, A., and Dammert, K.: Alkaline Phosphatase Activity in

Cholesteatoma Epithelium and Skin 153

Palva, A.: vide Palva, T.

Penders, C. et Boniver, R.: Exploration électrophysiologique du réflexe de

clignement dans la paralysie faciale a frigore 17

Pfaltz, C. R.: Editorial 193

Pfaltz, C. R.: vide M $\gamma y$ oshi, T.

Piffkó, P.; Köteles, G. I., and Antoni, F.: The Effect of Some Antibiotics on

Protein Synthesis in Human Tonsillar Cells, in vitro 10

Piffkó, P.: vide Puskás, M.

Pruszewicz, A.; Obrebowski, A.; Donat-Jasiak, T., and Szmejowa, A.: Radio-logic Picture of the

Hypopharynx Recesses after Laryngectomy .... 296

Puh $\lambda$ kka, H. J. and Hänninen, P.: Otolaryngological Findings in Patients with

Chronic Respiratory Paralysis after Poliomyelitis 220

Puskás, M.; Antoni, F.; Staub, M.; Gy. Farkas, and Piffkó, P.: Lysozyme

Activity of the Human Tonsil 160

Renner, K.: vide Kallenberger, Alice

Rivier, A.: vide Savary, M.

Savary, M. et Rivier, A.: Possibilités et limites de Гæsophagoscopie

Schätzle, W.: vide Miehlke, A.

Schnieder, E.-A.: Experiences with Tissue Adhesives in Middle Ear Operations 227

Schuster, R.: vide Miehlke, A. 
Secretan, J.-P.: La fonction labyrinthique chez Гinfirme moteur cerebral .... 111

Staub, M.: vide Puskás, M.

Stennert, E.: vide Miehlke, A.

Szmejowa, A.: vide Pruszewicz, A.

IV

Index

Westergaard, O.; Kluyskens, P.; John, H. D.: Alport's Syndrome. Histopathology of Human Temporal Bones

263

Wey, W.: vide Kallenberger, Alice

Withers, B. T.: vide Igarashi, M.

Youngs, J. N. and Juhn, S. K.: Perilymph Findings after Intravenous Urea

Administration 140

Society Transactions. Gesellschaftsberichte. Sociétés

58. Versammlung der Schweizerischen Gesellschaft für Oto-Rhino-Laryngologie,

Hals- und Gesichtschirurgie, Bürgenstock, 17.-19. Juni 1971

27

Nederlandse Keel-Neus-Oorheelkundige Vereniging. 133ste Vergadering te

Groningen, 30 oktober 1971 - Dutch Oto-Rhino-Laryngological So

ciety. 133rd Meeting, Groningen, 30th October $1971 \quad 176$

Nederlandse Keel-Neus-Oorheelkundige Vereniging. 134e Vergadering te Nij-

megen, 28 en 29 april 1972 - Dutch Oto-Rhino-Laryngological So

ciety. 134th Meeting, Nijmegen, April 28 and 29, $1972 \quad 331$

59. Frühjahrsversammlung der Schweizerischen Gesellschaft für Oto-Rhino-

Laryngologie, Hals- und Gesichtschirurgie, Locarno, 8.-10. Juni 1972356

Book Reviews - Buchbesprechungen - Livres nouveaux $\quad 64,124,191,259,372$

Varia 126, 324

Index rerum ad Vol. $34 \quad 374$

Index nominum ad Vol. $34 \quad 378$

S. Karger · Basel · München · Paris · London · New York · Sydney Arnold-Böcklin-Strasse 25, CH-4011 Basel (Switzerland)

All rights, including that of translation into other languages, reserved.

Photomechanic reproduction (photocopy, microcopy) of this volume or parts thereof

without special permission of the publishers is prohibited.

(C) Copyright 1972 by S. Karger AG, Verlag für Medizin und Naturwissenschaften, Basel Printed in Switzerland by Buchdruckerei Friedrich Reinhardt AG, Basel 